NASA/TM-2001-210063

\title{
An Analysis and Procedure for Determining Space Environmental Sink Temperatures With Selected Computational Results
}

Albert J. Juhasz

Glenn Research Center, Cleveland, Ohio 
Since its founding, NASA has been dedicated to the advancement of aeronautics and space science. The NASA Scientific and Technical Information (STI) Program Office plays a key part in helping NASA maintain this important role.

The NASA STI Program Office is operated by Langley Research Center, the Lead Center for NASA's scientific and technical information. The NASA STI Program Office provides access to the NASA STI Database, the largest collection of aeronautical and space science STI in the world. The Program Office is also NASA's institutional mechanism for disseminating the results of its research and development activities. These results are published by NASA in the NASA STI Report Series, which includes the following report types:

- TECHNICAL PUBLICATION. Reports of completed research or a major significant phase of research that present the results of NASA programs and include extensive data or theoretical analysis. Includes compilations of significant scientific and technical data and information deemed to be of continuing reference value. NASA's counterpart of peerreviewed formal professional papers but has less stringent limitations on manuscript length and extent of graphic presentations.

- TECHNICAL MEMORANDUM. Scientific and technical findings that are preliminary or of specialized interest, e.g., quick release reports, working papers, and bibliographies that contain minimal annotation. Does not contain extensive analysis.

- CONTRACTOR REPORT. Scientific and technical findings by NASA-sponsored contractors and grantees.
- CONFERENCE PUBLICATION. Collected papers from scientific and technical conferences, symposia, seminars, or other meetings sponsored or cosponsored by NASA.

- SPECIAL PUBLICATION. Scientific, technical, or historical information from NASA programs, projects, and missions, often concerned with subjects having substantial public interest.

- TECHNICAL TRANSLATION. Englishlanguage translations of foreign scientific and technical material pertinent to NASA's mission.

Specialized services that complement the STI Program Office's diverse offerings include creating custom thesauri, building customized data bases, organizing and publishing research results ... even providing videos.

For more information about the NASA STI Program Office, see the following:

- Access the NASA STI Program Home Page at http://www.sti.nasa.gov

- E-mail your question via the Internet to help@sti.nasa.gov

- Fax your question to the NASA Access Help Desk at 301-621-0134

- Telephone the NASA Access Help Desk at 301-621-0390

- Write to: NASA Access Help Desk NASA Center for AeroSpace Information 7121 Standard Drive Hanover, MD 21076 
NASA/TM-2001-210063

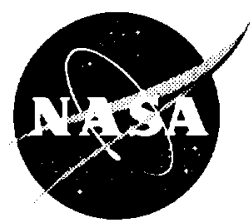

\title{
An Analysis and Procedure for Determining Space Environmental Sink Temperatures With Selected Computational Results
}

\author{
Albert J. Juhasz \\ Glenn Research Center, Cleveland, Ohio
}

Prepared for the

2000 International Conference on Environmental Systems sponsored by the Society of Automotive Engineers

Toulouse, France, July 11-14, 2000

National Aeronautics and

Space Administration

Glenn Research Center 
Available from

NASA Center for Aerospace Information 7121 Standard Drive

Hanover, MD 21076

Price Code: $\mathrm{A} 03$
National Technical Information Service 5285 Port Royal Road Springfield, VA 22100 Price Code: A03

Available electronically at http://gltrs.grc.nasa.gov/GLTRS 


\title{
AN ANALYSIS AND PROCEDURE FOR DETERMINING SPACE ENVIRONMENTAL SINK TEMPERATURES WITH SELECTED COMPUTATIONAL RESULTS
}

\author{
Albert J. Juhasz \\ National Aeronautics and Space Administration \\ Glenn Research Center \\ Cleveland, Ohio 44135
}

SUMMARY

\begin{abstract}
The purpose of this report was to analyze the heat-transfer problem posed by the determination of spacecraft temperatures and to incorporate the theoretically derived relationships in the computational code TSCALC. The basis for the code was a theoretical analysis of the thermal radiative equilibrium in space, particularly in the Solar System. Beginning with the solar luminosity, the code takes into account these key variables: the spacecraft-to-Sun distance expressed in astronomical units (AU), where $1 \mathrm{AU}$ represents the average Sun-to-Earth distance of 149.6 million $\mathrm{km}$; the angle (arc degrees) at which solar radiation is incident upon a spacecraft surface (ILUMANG); the spacecraft surface temperature (a radiator or photovoltaic array) in kelvin; the surface absorptivity-to-emissivity ratio $\alpha / \varepsilon$ with respect to the solar radiation and $(\alpha / \varepsilon)_{2}$ with respect to planetary radiation; and the surface view factor to space $F$.
\end{abstract}

\section{INTRODUCTION}

For the thermal design of spacecraft radiators, it is necessary to determine a space background temperature, or equivalent space sink temperature, to which radiators reject their design heat load. This is especially true for radiators that operate at the relatively low temperature range of 300 to $500 \mathrm{~K}$, for which early assumptions of 0 to $3 \mathrm{~K}$ for the sink temperature introduced serious errors in the determination of the required radiator area. This equivalent space sink temperature is not the temperature of the vacuum surrounding a spacecraft; rather, it is an equilibrium temperature that a passive radiating surface assumes by exchanging thermal radiation energy with the space environment without any onboard (internally generated) heat load that has to be rejected to space. This equilibrium temperature, a function of the spacecraft-to-Sun distance and the angle at which the surface intercepts solar radiation, is greatly influenced by the characteristics (key variables) of the radiating surface. These are the view factor to space $F$ and the $\alpha / \varepsilon$ ratio, which expresses the surface absorptivity of solar radiation to the emissivity at its final equilibrium temperature when heat is radiated to space, usually in the infrared region of the spectrum.

Early reports (Goldman and Singer, 1957) assumed for radiator surfaces gray-body characteristics that gave $\alpha / \varepsilon$ values near unity. The work of Rittenhouse and Singletary (1968) showed that special metal oxide coatings such as Z-93 could be applied to surfaces to lower the amount of solar energy absorbed while keeping infrared emissivity high. With the achievement of $\alpha / \varepsilon$ values near 0.1 for these coatings, the space equivalent sink temperatures have been effectively lowered, permitting more heat to be rejected by a radiator with a given surface area and an effective radiating temperature.

The purpose of this report was to derive the equilibrium temperatures that actual spacecraft radiating surfaces would experience from the combined effects of solar and planetary radiation and to determine the amount of onboard heat to be rejected. The derivation is based on a new definition of the term "space sink temperature" and differs from earlier definitions, such as that of Gordon (1982), who defined the temperature of space as the equilibrium temperature that a small black sphere would experience.

In contrast to Gordon's (1982) definition of the space sink temperature, the theoretical derivations in this report are based on the equilibrium temperature that a radiating surface having a given $\alpha / \varepsilon$ ratio would achieve in space. The conditions are that the surface does not have internal heat to be rejected but is exposed to a given incident radiant energy flux from the Sun (or star) and planetary surfaces, part of which is reradiated to the space background. The amount of thermal energy that reradiates into space is determined by the key variables, such as the $\alpha / \varepsilon$ ratio and the view factor to space. Two additional statements can be made regarding the authors' definition of space sink temperature: 
1. If the incident radiant energy flux is reduced to zero, the equilibrium sink temperature for a surface without internal heat generation would drop to $0 \mathrm{~K}$. Note, however, that this condition cannot be realistically achieved, even in interstellar space where thermal equilibrium is attained at about $3 \mathrm{~K}$.

2. For a radiating surface that is rejecting an internal heat load, the average equilibrium surface temperature increases to a value consistent with the higher temperature value in the Stefan-Boltzmann equation. Substituting the sink temperature, as expressed by the new definition, for the lower temperature value in the same equation results in the radiated heat being equal to the internal heat load that must be rejected by the surface.

\section{SYMBOLS}

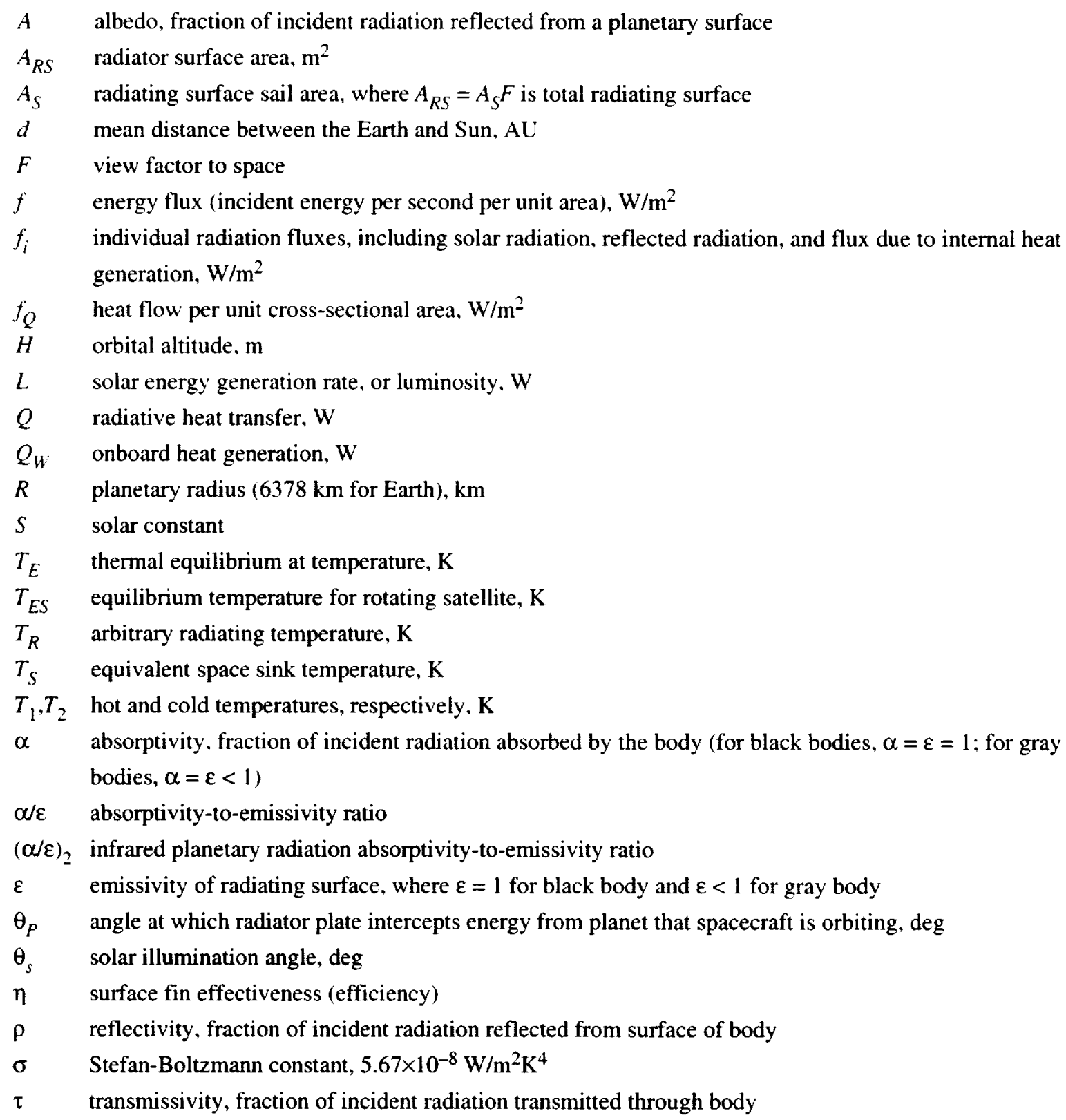




\section{ANALYSIS OF RADIATION HEAT TRANSFER IN SPACE}

This section presents a mathematical analysis of radiation heat transfer in space. The derivation of the constitutive equations and relationships that describe radiant energy exchange in space has been used as the basis for developing the computational code TSCALC.

\section{Derivation of Equations}

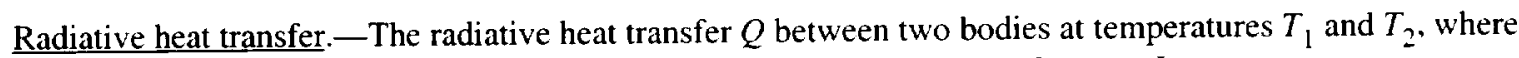
$T_{2} \ll T_{1}$ (negligible reradiation), can be expressed according to the Stefan-Boltzmann Law as

$$
Q=\sigma \varepsilon F A_{s}\left(T_{1}^{4}-T_{2}^{4}\right)
$$

where

$Q \quad$ radiated heat flow, $\mathrm{W}$

$\sigma \quad$ Stefan-Boltzmann constant, $5.67 \times 10^{-8} \mathrm{~W} / \mathrm{m}^{2} \mathrm{~K}^{4}$

$\varepsilon \quad$ emissivity of radiating surface, where $\varepsilon=1$ for black body and $\varepsilon<1$ for gray body

$F \quad$ surface area view factor between radiating bodies

$A_{R S} \quad$ radiating surface sail area, $\mathrm{m}^{2}$

$A_{S} \quad$ radiating surface sail area, where radiator surface area $A_{R S}=A_{S} F$ is total radiating surface

$T_{1}, T_{2}$ hot and cold temperatures, respectively, $\mathrm{K}$

Objects in the vacuum of space at arbitrary radiating temperatures $T_{R}$ lose heat by radiation to an environmental equilibrium, or space sink temperature $T_{S}$, that is very near absolute zero $(\sim 3 \mathrm{~K})$ in interstellar space. However, as shown later in the Results and Discussion section, $T_{S}$ can be several hundred degrees kelvin in the neighborhood of the inner planets of the Solar System. Hence, to correctly size the areas for space satellite radiators that operate at temperatures between 300 and $400 \mathrm{~K}$, the equivalent space sink temperatures must be determined within an accuracy of a few tens of degrees.

For the space radiation case, equation (1) can be rewritten in terms of $T_{R}$ and $T_{S}$ :

$$
Q=\sigma \varepsilon F A_{s}\left(T_{R}^{4}-T_{S}^{4}\right)
$$

The equivalent space sink temperatures $T_{S}$ in the neighborhood of planets in the Solar System was calculated as follows. Starting with the solar energy generation rate (or luminosity) $L$, the energy absorption rate per Earth (or planet) unit surface area needs to be balanced by the re-emitted energy rate for thermal equilibrium to be achieved at $T_{E}$ or $T_{\text {EARTH }}$. For this analysis, $F$ is set to unity. If the Sun is represented as a heat source at the center of a sphere whose radius is equal to the mean distance between the Earth and Sun $d$, the figure shows that about $1370 \mathrm{~W}$ fall on each square meter that is perpendicular to the radiating energy flux. The value $1370 \mathrm{~W} / \mathrm{m}^{2}$ is referred to as the solar constant at $1 \mathrm{AU}$ (astronomical unit), where $d=1.496 \times 10^{11} \mathrm{~m}$. The value of the solar constant $S$ can be determined by dividing the luminosity $L$ of the Sun by the area of the sphere with a radius equal to $1 \mathrm{AU}$ :

$$
S=\frac{L}{4 \pi d^{2}}
$$

Substituting $L$ with the value of $3.86 \times 10^{26} \mathrm{~W}$ gives

$$
S=1372.5 \mathrm{~W} / \mathrm{m}^{2} \quad \text { for } 1 \mathrm{AU}
$$

and when rounded to three significant figures becomes

$$
S=1370 \mathrm{~W} / \mathrm{m}^{2}
$$




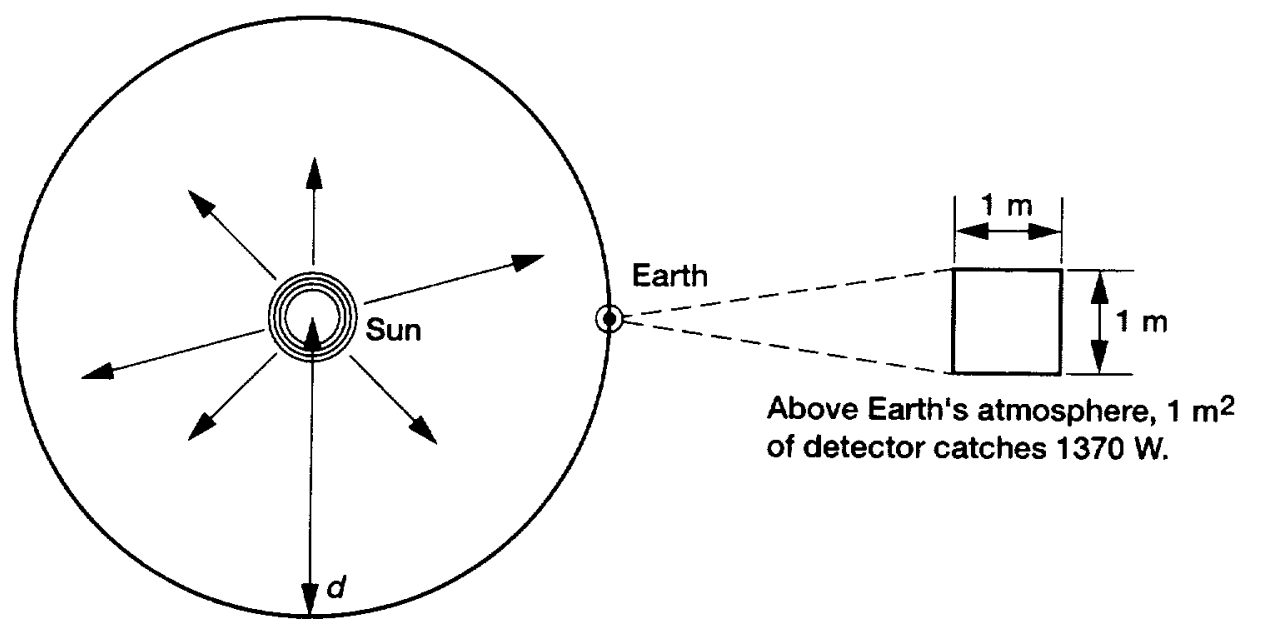

A giant sphere, $1 \mathrm{AU}$ in radius, would catch all the Sun's radiative energy.

Figure 1.-Sun-to-Earth heat radiation case.

Note that the result shown in equation (3c) represents the incident energy rate per unit Earth surface area that is perpendicular to the solar energy flux. Before we determine the fraction of this energy flux absorbed by the surface, some important relationships must be reviewed in the next section.

Absorption, reflection, and transmission of radiation.- When electromagnetic radiation impinges on a body, it is partially absorbed, partially reflected, and partially transmitted. The relation between the absorbed, reflected, and transmitted energy is

where

$$
\alpha+\rho+\tau=1
$$

$\alpha$ absorptivity, fraction of incident radiation absorbed by the body (for black bodies, $\alpha=\varepsilon=1$; for gray bodies, $\alpha=\varepsilon<1$ )

$\rho$ reflectivity, fraction of incident radiation reflected from surface of body

$\tau$ transmissivity, fraction of incident radiation transmitted through body

For opaque bodies such as the planets, transmissivity $\tau=0$. Equation (4) then can be expressed as

$$
\alpha+\rho=1
$$

\section{Calculation of Planetary Temperatures in Solar System}

Space radiators in planetary orbits are exposed to solar and planetary radiation; therefore, we must first determine the equilibrium temperatures at which planets radiate to spacecraft, even if the spacecraft is in the planetary shadow and does not receive any solar radiation.

Planets also reflect part of the incident solar radiation into space where orbiting spacecraft may receive some of it in addition to the direct radiation heating by the Sun. The reflectivity of planets is referred to as the albedo and is represented by $A$ in equation ( $5 b$ ). Thus, from equation ( $5 a$ ), the absorptivity can be expressed as

$$
\alpha=1-A
$$

To proceed with the calculation of equivalent space sink temperature $T_{S}$ at a given planetary orbit distance from the Sun, the energy flux, or incident energy per second per unit area $f$, is defined as 


$$
f=\frac{\text { Energy rate }(\mathrm{W})}{\text { Area }\left(\mathrm{m}^{2}\right)}
$$

For thermal equilibrium at temperature $T_{E}$ to be achieved,

$$
f_{\text {emitted }} \times \text { Radiating area }=f_{\text {absorbed }} \times \text { Absorbing area }
$$

where (fig. 1)

$$
f_{\text {emitted }}=\sigma \varepsilon T_{E}^{4} \quad \text { and } \quad f_{\text {absorbed }}=\frac{(1-A) L}{4 \pi d^{2}}
$$

Because the Earth is a rapidly rotating planet, once every $24 \mathrm{hr}$, its entire surface is considered to be at approximately the same temperature. Hence, the total surface energy (or heat) emitted will be

$$
f_{\text {emitted }} \times 4 \pi R^{2}
$$

where $R$ is the planetary radius ( $6378 \mathrm{~km}$ for the Earth).

The total heat intercepted by the Earth from the Sun is the heat flux times the projected Earth area on an imaginary sphere with a radius of $1 \mathrm{AU}$. This area is represented as $\pi R^{2}$. Thus, the total energy absorbed is $f_{\text {absorbed }} \times \pi R^{2}$.

Substitute the expressions from equation (7) to obtain

$$
\sigma \varepsilon T_{E}^{4} \times 4 \pi R^{2}=\frac{(1-A) L \times \pi R^{2}}{4 \pi d^{2}}
$$

Solving equation (9) for $T_{E}$, one obtains

$$
T_{E}=\sqrt[4]{\frac{(1-A) L}{16 \pi \sigma \varepsilon d^{2}}}
$$

Substituting the numerical values for $L, d$, and $\sigma$ yields, after some simplification.

$$
T_{E}=279 \sqrt[4]{\frac{(1-A)}{\varepsilon d^{2}}}
$$

where $T_{E}$ is in kelvin and $d$ is in astronomical units (Earth $=1 \mathrm{AU}$ ).

If the approximate value of the Earth's albedo $(A \sim 0.3)$ and the emissivity $(\varepsilon \sim 0.8)$ are substituted in equation (11), we obtain the approximate value of $270 \mathrm{~K}$ for $T_{E}$, which will be used to calculate the radiation from Earth to the orbiting spacecraft.

Recall that equation (11) was derived for rapidly rotating planets such as Earth or Mars. For slowly rotating planets like Mercury and the Moon, one must take into account that these bodies receive energy over their projected (disk) areas and emit energy, not over their full spherical surface areas but only over the same projected areas because the remaining surface area is considered to be too cold to radiate a significant amount of energy back to space. For such bodies, the thermal equilibrium is thus established when

$$
\sigma \varepsilon T_{E}^{4} \times \pi R^{2}=\frac{(1-A) L \times \pi R^{2}}{4 \pi d^{2}}
$$


and

$$
T_{E}=\sqrt[4]{\frac{(1-A) L}{4 \pi \sigma \varepsilon d^{2}}}
$$

or

$$
T_{E}=394 \sqrt[4]{\frac{(1-A)}{\varepsilon d^{2}}}
$$

where, as before, the Sun-Earth distance $d$ is expressed in AU. A comparison of equations (10) and (13a) shows that for slowly rotating planets, the equilibrium temperature is higher by a factor equivalent to the fourth root of the projected area (i.e., the ratio of sphere surface-to-disk area), namely the fourth root of 4 .

Apply equation (13a), for $d=1 \mathrm{AU}, \varepsilon=1$, and $A=0.07$, to Earth's moon to obtain

$$
T_{E}=394 \sqrt[4]{\frac{(1-0.07)}{1^{2}}}=387 \mathrm{~K}
$$

which is the maximum temperature at the lunar equator at noon. Note that on the dark side (during the 356-hr lunar night), the temperature is $\sim 70 \mathrm{~K}$.

\section{Calculation of Spacecraft Temperatures in Planetary Orbits}

In addition to the electromagnetic radiation from the solar spectrum, spacecraft in planetary orbits receive longwavelength infrared radiation from planets they orbit.

Near-spherical satellites.-For polyhedral spacecraft geometries that are nearly spherical, equation (10) will give a good approximation of the equilibrium temperature due to solar radiation. However, the term that expresses the absorptivity-to-emissivity ratio for the planetary surface $(1-A) / \varepsilon$ is replaced by the absorptivity-to-emissivity ratio $\alpha / \varepsilon$ for the radiating surface material. Thus, for near-spherical shapes, the equilibrium temperature for the rotating satellite $T_{E S}$ becomes

$$
T_{E S}=\sqrt[4]{\frac{\left(\frac{\alpha}{\varepsilon}\right)\left(\frac{1}{F}\right) L}{16 \pi \sigma d^{2}}}
$$

where the value of the view factor to space $F$ will normally be close to unity but can be lower if the radiating surface is partially occulted by projecting parts of the spacecraft. Lowering the value of $F$ will cause $T_{E S}$ to increase.

Flat-plate radiator surfaces.-For flat-plate radiators, in addition to the $\alpha / \varepsilon$ ratio, we need to include the solar illumination angle $\theta_{S}$, the angle at which the radiator plate intercepts the solar flux. Thus, the equilibrium temperature $T_{E}$ due to reflected solar radiation can be expressed as

$$
T_{E}=\sqrt[4]{\frac{A L \sin \theta_{S}\left(\frac{\alpha}{\varepsilon}\right) \tau}{F\left(4 \pi \sigma d^{2}\right)}}
$$

where $\tau$ is the transmissivity of the atmosphere. If a satellite is outside a planetary atmosphere, as is normally the case, $\tau$ is set to unity. 
Albedo.-Recall that the albedo $A$ is the fraction of incident radiation reflected from a planetary surface: thus. for a surface in Earth orbit at altitude $H$, we have from equation ( 8 a)

$$
f_{\text {reflected }}=\left(\frac{A L \tau}{4 \pi d^{2}}\right) \times\left(\frac{R_{E}}{R_{E}+H}\right)^{2}
$$

where $f_{\text {reflected }}$ is the reflected radiation energy flux per unit area at orbital altitude $H$ (in meters), and $R_{E}$ is the radius of the Earth $\left(6.378 \times 10^{6} \mathrm{~m}\right)$. Note that the atmospheric transmissivity $(\tau<1)$ reduces the reflected radiation flux. Of this energy flux, the fraction absorbed by a radiating surface is

$$
f_{\text {absorbed }}=f_{\text {reflected }} \sin \theta_{P} \alpha
$$

where $\theta_{P}$ is the angle at which the radiator plate intercepts energy from a planet that the spacecraft is orbiting.

Therefore, due to albedo alone, the equilibrium temperature of a satellite's radiating surface is

$$
T_{E}=\sqrt[4]{\frac{A L \tau \sin \theta_{p}\left(\frac{\alpha}{\varepsilon}\right)}{F\left(4 \pi \sigma d^{2}\right)}\left(\frac{R_{E}}{R_{E}+H}\right)^{2}}
$$

Earth shine, or direct planetary radiation.-For direct radiation from Earth or a planet at its previously determined equilibrium temperature $T_{\mathrm{EARTH}}$, the radiation heat flux at or near the planetary surface is

$$
f_{\text {radiated }}=\sigma \varepsilon \tau T_{\mathrm{EARTH}}^{4}
$$

and the equilibrium temperature $T_{\mathrm{E}}$ of a radiating surface at orbital altitude $H$, due to direct Earth (planet) shine, is given by

$$
T_{E}=\sqrt[4]{\left(\frac{f_{\text {radiated }}}{F}\right)\left[\left(\frac{\alpha}{\varepsilon}\right)_{2} \tau \sin \theta_{P}\left(\frac{1}{\sigma}\right)\right]\left(\frac{R_{E}}{R_{E}+H}\right)^{2}}
$$

where $(\alpha / \varepsilon)_{2}$ is the infrared planetary radiation absorptivity-to-emissivity ratio of the plate radiator surface and $\tau$ is the transmissivity of the atmosphere. In most cases, $(\alpha / \varepsilon)_{2}$ will be close to unity.

Internal heat generation.- A spacecraft radiator surface normally rejects heat generated onboard. This rejection causes a rise in the equilibrium radiating temperature because of the internal heat flux (the heat flow per unit crosssectional area $f_{Q}$ ):

$$
f_{Q}=\frac{Q_{W}}{F \varepsilon \eta A_{R S}}
$$

where $\eta$ is the surface fin effectiveness (efficiency) and $Q_{W}$ is the onboard heat generation in watts. In the absence of all other effects, this equilibrium temperature would be represented by

$$
T_{E}=\sqrt[4]{\frac{Q_{W}}{F \varepsilon \sigma \eta A_{R S}}}
$$


Combined effects.- To determine the combined effects on $T_{E}$ of the heat-transfer mechanisms discussed in the previous sections, the individual radiation fluxes $f_{i}$ are added and set equal to $\sigma \varepsilon T_{E}^{4}$. When solved for $T_{E}$, the following equation expresses the combined effects of solar radiation, albedo, planetary surface temperature radiation in the long-wavelength infrared region, and internal heat generation:

$$
T_{E}=\sqrt[4]{\frac{\sum f_{i}}{F(\sigma \varepsilon)}}
$$

\section{RESULTS AND DISCUSSION}

The equations expressing the thermal equilibrium relationships derived in the section Analysis of Radiation Heat Transfer in Space were incorporated in the computer code TSCALC. Several options in this code permit the user to evaluate the effects of key input variables, such as solar distance $d(\mathrm{AU})$, the incident angles for solar and planetary radiation $\theta_{S}$ (ILUMANG) and $\theta_{P}$ (ILUM2), the absorptivity-to-emissivity ratio $\alpha / \varepsilon$ (AE) for solar radiation, the infrared planetary radiation absorptivity-to-emissivity ratio $(\alpha / \varepsilon)_{2}(\mathrm{AE} 2)$, and the view factor to space $F$ (FV). Note that FV can have a value up to 2 for flat-plate radiators rejecting heat from both sides. If both sides do not have a full $2 \pi$ steradian view of space, the value will be less than 2 . For a sphere that receives thermal radiation over its projected area but reradiates over the full surface area. FV can have a maximum value of 4 .

An example showing the increase in equilibrium temperatures for a spacecraft surface approaching the Sun from the heliopause at $220 \mathrm{AU}$ is shown in table I. Note that the corona temperature of nearly $2000 \mathrm{~K}$ at the $0.02-\mathrm{AU}$ position represents the expected value at the closest approach during the future perihelion mission. A carbon-carbon heat shield having an $\alpha / \varepsilon$ ( $\mathrm{AE}$ ) of 0.6 and intercepting solar radiation at $25^{\circ}$ (ILUMANG) may be used to shield the spacecraft.

Figure 2 graphically depicts the heat flux and temperature information. Note that the temperatures shown are for planetary orbit distances, not for the planets themselves. However, planetary temperatures could be computed by the code if the proper input values (ILUMANG, FV, and AE) were used.

Table II shows the effect at $1 \mathrm{AU}$ of the insolation angle, which is defined as the angle at which a reradiating surface without internal heat generation $\left(Q_{W}=0\right)$ intercepts the solar energy flux. Note that for a solar incident angle $\theta_{S}$ of $0^{\circ}$. the code gives a value of $3 \mathrm{~K}$ because the solar heat flux has been eliminated and the equilibrium temperature is that of interstellar space.

The information in table III can be used to consider the example of equilibrium temperatures determined primarily by planetary radiation in the infrared domain for a space structure in geostationary orbit at a $35876-\mathrm{km}$ altitude. By setting the incident angle for solar radiation (ILUMANG) equal to $0.1^{\circ}$, we assume that 99.9 percent of the solar heat flux can be eliminated by shielding and insulation. Also, by assuming an Earth-gravity-gradient-stabilized structure, one can see how the space sink temperature varies as the direct radiation from the Earth is incident upon a surface from $1^{\circ}$ to $10^{\circ}$ of arc. From the table, we can see that temperatures lower than $77 \mathrm{~K}$ (liquid nitrogen boiling point at atmospheric pressure) can be maintained if the angle of incident Earth radiation can be held to less than $5^{\circ}$.

\begin{tabular}{|c|c|c|c|c|c|c|c|}
\hline $\begin{array}{c}\text { ILUMANG } \\
\text { (DEG) }\end{array}$ & FV & EPS & $\mathrm{AE}$ & $\mathrm{AU}$ & $\begin{array}{l}\text { Q/A(W/M2) } \\
\text { (90 DEG.) }\end{array}$ & $\mathrm{TS}(\mathrm{K})$ & ORBIT \\
\hline 25.00 & 1.0 & .88 & .60 & 220.000 & .03 & 18.9 & HLPAUSE \\
\hline 25.00 & 1.0 & .88 & .60 & 39.438 & .88 & 44.6 & PLUTO \\
\hline 25.00 & 1.0 & .88 & .60 & 30.058 & 1.52 & 51.1 & NEPTUNE \\
\hline 25.00 & 1.0 & .88 & .60 & 19.182 & 3.73 & 63.9 & URANUS \\
\hline 25.00 & 1.0 & .88 & .60 & 9.539 & 15.08 & 90.6 & SATURN \\
\hline 25.00 & 1.0 & .88 & .60 & 5.203 & 50.70 & 122.7 & JUPITER \\
\hline 25.00 & 1.0 & .88 & .60 & 3.000 & 152.50 & 161.6 & ASTRDS \\
\hline 25.00 & 1.0 & .88 & .60 & 1.524 & 591.18 & 226.8 & MARS \\
\hline 25.00 & 1.0 & .88 & .60 & 1.000 & 1372.51 & 279.9 & EARTH \\
\hline \multirow[t]{2}{*}{25.00} & 1.0 & & .60 & .723 & 2623.26 & 329.1 & VENUS \\
\hline & & .88 & & & & & \\
\hline 25.00 & 1.0 & .88 & .60 & .387 & 9164.15 & 450.0 & MERCURY \\
\hline 25.00 & 1.0 & .88 & .60 & .020 & 3431265.02 & 1979.3 & CORONA \\
\hline
\end{tabular}




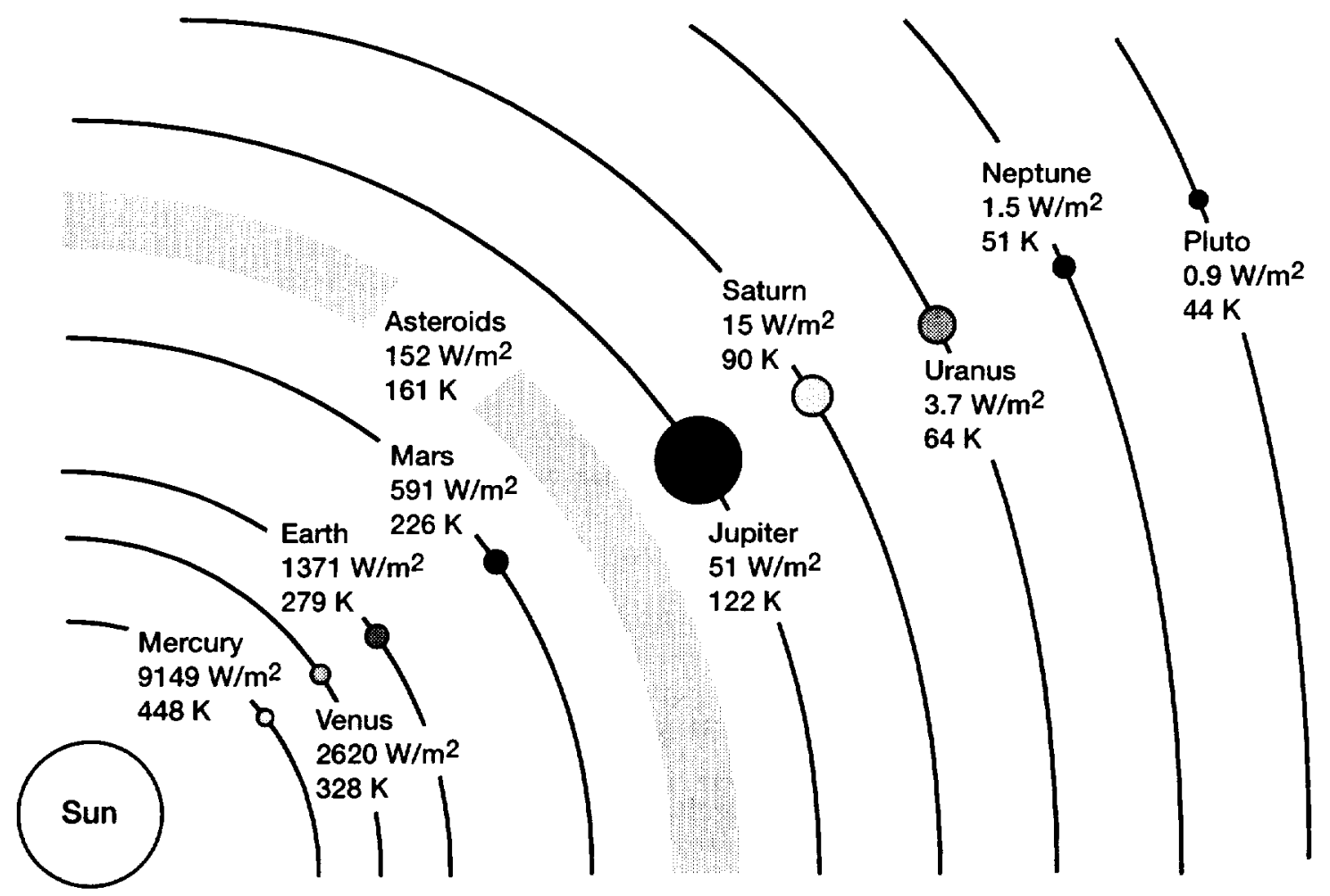

Figure 2.-Solar heat flux and spacecraft surface temperatures at various planetary orbit distances.

TABLE II.-SINK TEMPERATURE (TS) AS FUNCTION OF INSOLATION ANGLE AT 1 AU

\begin{tabular}{|rrrrrrr|}
\hline $\begin{array}{r}\text { IUMANG } \\
\text { (DEG) }\end{array}$ & FV & EPS & AE & AU & TS(K) & QW-WATTS \\
.00 & 1.5 & .88 & .18 & 1.000 & 3.0 & .0 \\
1.00 & 1.5 & .88 & .18 & 1.000 & 84.4 & .0 \\
2.00 & 1.5 & .88 & .18 & 1.000 & 100.3 & .0 \\
3.00 & 1.5 & .88 & .18 & 1.000 & 111.0 & .0 \\
4.00 & 1.5 & .88 & .18 & 1.000 & 119.3 & .0 \\
& & & & & & .0 \\
5.00 & 1.5 & .88 & .18 & 1.000 & 126.1 & .0 \\
10.00 & 1.5 & .88 & .18 & 1.000 & 149.9 & .0 \\
15.00 & 1.5 & .88 & .18 & 1.000 & 165.6 & .0 \\
20.00 & 1.5 & .88 & .18 & 1.000 & 177.5 & .0 \\
25.00 & 1.5 & .88 & .18 & 1.000 & 187.2 & .0 \\
30.00 & 1.5 & .88 & .18 & 1.000 & 195.2 & .0 \\
35.00 & 1.5 & .88 & .18 & 1.000 & 202.0 & .0 \\
40.00 & 1.5 & .88 & .18 & 1.000 & 207.9 & .0 \\
45.00 & 1.5 & .88 & .18 & 1.000 & 212.9 & .0 \\
50.00 & 1.5 & .88 & .18 & 1.000 & 217.2 & .0 \\
55.00 & 1.5 & .88 & .18 & 1.000 & 220.9 & .0 \\
60.00 & 1.5 & .88 & .18 & 1.000 & 224.0 & .0 \\
90.00 & 1.5 & .88 & .18 & 1.000 & 232.2 & \\
\hline
\end{tabular}


TABLE III.-GEOSTATIONARY ORBIT EQUILIBRIUM TEMPERATURE AT NEAR ECLIPSE

\begin{tabular}{|c|c|c|c|c|c|c|c|c|c|}
\hline \multicolumn{2}{|c|}{ ILUMANG (DEG) } & ALTITUDE & FV & TR-K & TS-K & $\overline{\mathrm{AE}}$ & $\overline{\mathrm{AE}-2}$ & $\overline{A U}$ & QW-WATTS \\
\hline Sun & Earth & $(\mathrm{K}-\mathrm{KM})$ & & & & & & & \\
\hline .1 & 1.0 & 35.88 & 1.0 & 300.0 & 59.5 & .18 & .80 & 1.000 & .0 \\
\hline .1 & 2.0 & 35.88 & 1.0 & 300.0 & 64.6 & .18 & .80 & 1.000 & .0 \\
\hline .1 & 3.0 & 35.88 & 1.0 & 300.0 & 68.7 & .18 & .80 & 1.000 & .0 \\
\hline .1 & 4.0 & 35.88 & 1.0 & 300.0 & 72.2 & .18 & .80 & 1.000 & .0 \\
\hline .1 & 5.0 & 35.88 & 1.0 & 300.0 & 75.2 & .18 & .80 & 1.000 & .0 \\
\hline .1 & 6.0 & 35.88 & 1.0 & 300.0 & 77.9 & .18 & .80 & 1.000 & .0 \\
\hline .1 & 7.0 & 35.88 & 1.0 & 300.0 & 80.4 & .18 & .80 & 1.000 & .0 \\
\hline .1 & 8.0 & 35.88 & 1.0 & 300.0 & 82.6 & .18 & .80 & 1.000 & .0 \\
\hline .1 & 9.0 & 35.88 & 1.0 & 300.0 & 84.7 & .18 & .80 & 1.000 & .0 \\
\hline .1 & 10.0 & 35.88 & 1.0 & 300.0 & 86.6 & .18 & .80 & 1.000 & .0 \\
\hline
\end{tabular}

Results of the type illustrated above have been used in the design of radiators for deep space probes (Juhasz, et al., 1999). The computational code can also be used to establish environmental conditions for structures that generate solar power in geostationary orbits.

\section{CONCLUDING REMARKS}

A detailed derivation of the constitutive equations and relationships that describe radiant energy exchange in space has been completed and used as the basis for developing a computational code. The equations describe heattransfer mechanisms related to key variables such as solar or stellar radiation, reflected planetary radiation (albedo), direct planetary radiation in the infrared portion of the electromagnetic spectrum, and onboard generated heat that must be rejected to the space environment.

To rapidly evaluate a combination of these heat-transfer mechanisms, the comprehensive multioptional computer code TSCALC was developed and verified against some independent determinations and measurements found in the literature.

Outputs from the code have also been used to determine equilibrium space sink temperatures for deep-space probes.

\section{REFERENCES}

Goldman, D.T.; and Singer, S.F.: Studies of a Minimum Orbital Unmanned Satellite of the Earth (MOUSE). Part III: Radiation Equilibrium and Temperature, Astronau. Acta, vol. 3, 1957, pp. 110-129.

Gordon, Gary D.: Spacecraft Technology. Part II: chapter 7-Thermal Control. Comsat Laboratories, March 1982, pp. $7-1$ to $7-44$.

Juhasz, Albert J.; Tew, Roy C.; and Thieme, Lanny G.: Design and Analysis Code for Heat Pipe Radiators of Stirling Power Systems Applicable to Deep Space Probes. Presented at the 11 th International Heat Pipe Conference, (Tokyo, Japan), Sept. 1999.

Rittenhouse, John B.; and Singletary, John B.: Space Materials Handbook: Supplement 2 to the Second Edition Space Materials Experience. Lockheed Tech. Report AFML-TR-64-40, Supp. 2, 1968. 


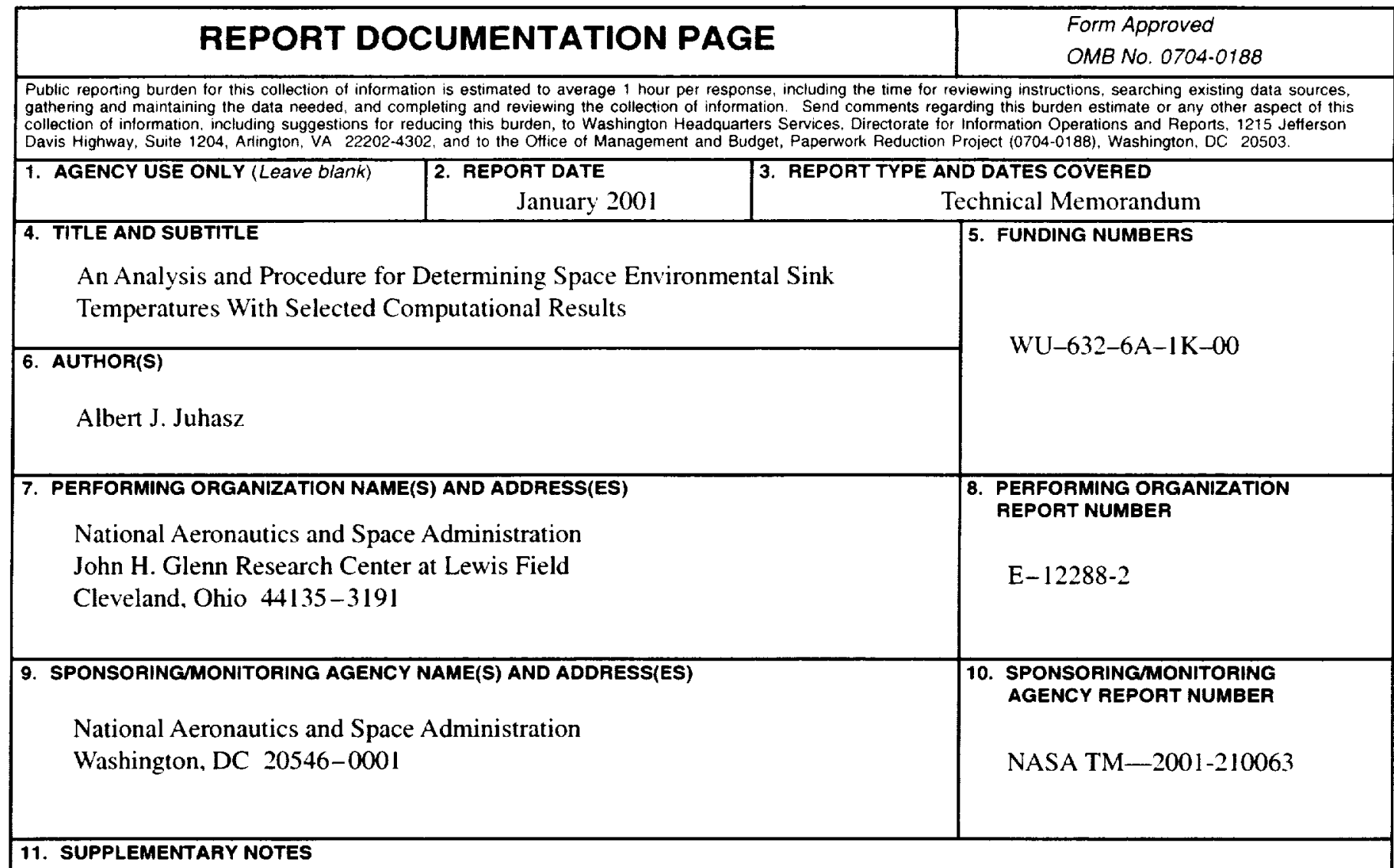

Prepared for the 2000 International Conference on Environmental Systems sponsored by the Society of Automotive Engineers, Toulouse, France, July 11-14, 2000. Responsible person, Albert J. Juhasz, organization code 5440,

$216-433-6134$.

12a. DISTRIBUTION/AVAILABILITY STATEMENT 12b. DISTRIBUTION CODE

Unclassified - Unlimited

Subject Categories: 18 and 64

Distribution: Standard

Available electronically at hitp://gltrs.gtc.nasa.gov/GLTRS

This publication is available from the NASA Center for AeroSpace Information. 301-621-0390.

13. ABSTRACT (Maximum 200 words)

The purpose of this report was to analyze the heat-transfer problem posed by the determination of spacecraft temperatures and to incorporate the theoretically derived relationships in the computational code TSCALC. The basis for the code was a theoretical analysis of the thermal radiative equilibrium in space, particularly in the Solar System. Beginning with the solar luminosity, the code takes into account these key variables: the spacecraft-to-Sun distance expressed in astronomical units (AU), where $1 \mathrm{AU}$ represents the average Sun-to-Earth distance of 149.6 million $\mathrm{km}$; the angle (arc degrees) at which solar radiation is incident upon a spacecraft surface (ILUMANG); the spacecraft surface temperature (a radiator or photovoltaic array) in kelvin; the surface absorptivity-to-emissivity ratio $\alpha / \varepsilon$ with respect to the solar radiation and $(\alpha / \varepsilon)_{2}$ with respect to planetary radiation; and the surface view factor to space $F$. Outputs from the code have been used to determine environmental temperatures in various Earth orbits. The code was also utilized as a subprogram in the design of power system radiators for deep-space probes.

14. SUBJECT TERMS

Space temperatures; Computer code

\begin{tabular}{|c|c|c|}
\hline $\begin{array}{c}\text { 17. SECURITY CLASSIFICATION } \\
\text { OF REPORT } \\
\text { Unclassified }\end{array}$ & $\begin{array}{c}\text { 18. SECURITY CLASSIFICATION } \\
\text { OF THIS PAGE } \\
\text { Unclassified }\end{array}$ & $\begin{array}{c}\text { 19. SECUAITY CLASSIFICATION } \\
\text { OF ABSTAACT } \\
\text { Unclassified }\end{array}$ \\
\hline
\end{tabular}


